\title{
Mutations in Duchenne and Becker Muscular Dystrophy Patients
}

\author{
Haghshenas M*, Azimian H, Zare Karizi SH \\ Hanieh Azimian- moghadam, Department of Genetics, Faculty of Science, Science And Research Branch, \\ Islamic Azad University Tehran, Iran (hanie.azimian@yahoo.com) \\ Maryam Haghshenas, Department of Biology, Faculty of Science, University of Guilan, Rasht, Iran \\ (e-mail: m_haghshenass@yahoo.com) \\ Hamid Mahmoudzaeh-Niknam* (Corresponding author), Department of Immunology, Pasteur Institute of Iran, \\ Tehran 13164, Iran (mahmoudh@pasteur.ac.ir)
}

\begin{abstract}
Duchenne and Becker muscular dystrophies are recessive devastating neuromuscular disorders linked to the X-chromosome. This short review aims to explain most important mutatios existing in Duchenne and Becker muscular dystrophy patients.
\end{abstract}

Keywords: Duchenne muscular dystrophy, Becker muscular dystrophy, Gene mutations.

\section{Introduction}

Duchenne and Becker muscular dystrophies (DMD and BMD) are recessive devastating neuromuscular disorders linked to the X-chromosome which is estimated to affects 1 in 3600 and 30000 newborn male, respectively [1].

Most DMD patients are diagnosed between 4 and 5 years of age .They then characterized by weakness in the proximal muscle, abnormal gait, calf hypertrophy, and also expressed Gowers' sign upon getting up [2].

Children with DMD typically become wheelchair depend by the age of 8-14 years old [3].

Respiratory insufficiency and cardiomyopathy gradually develops. Without intervention and multidisciplinary, patients usually succumb to the disorder in their early twenties [4].

BMD is a milder form of disease with a later onset of symptoms and longer duration of survival. In BMD patients, wheelchair confinement typically postponed after 16 [5]

Both DMD and BMD are caused by a mutation in the DMD gene which spans $2.4 \mathrm{mb}$ at Xp21.2 and encode dystrophin protein [6]

Dystrophin consists of four different functional domains and involved with the dystrophin-associated glycol protein complex (DGC) at the inner surface of muscle fiber and anchors muscle cell membrane to the extra cellular matrix [7]

DMD is associated with greater than 4000 mutations in DMD gene. These mutations incorporated of deletions (about 60\%), as well as duplications (5-15\%), and small mutations (25-35\%) [8].

DMD deletion mutations cluster into two hotspots within exons 44-55 and 3-17, duplications occur mostly at the 5'-end of the gene and small mutations are distributed throughout the whole dystrophin gene. When the DMD mutation is in-frame and a semi functional protein is produced, patients are likely to develop BMD, whereas out of frame mutations disrupt the gene and cause the sever form of disease [9].

The ratio of male and female mutation rates in Duchenne muscular dystrophy, although overall they appear to be about equal, differ depending on the type of mutation present. In presumed point mutations, error in spermatogenesis are the most likely cause of the mutations while the opposite appears true in deletions. Only about $13 \%$ of deletions arise in spermatogenesis and $87 \%$ occur in oogenesis. $95 \%$ of point mutations arise in spermatogenesis while only about $5 \%$ results from an event in oogenesis [10]. 
Spontaneous mutations are responsible for about one-third of the affected boys [8].

Over the past decade there have been significant advances in the diagnostic approach to dystrophinopathy. Until 1988, the diagnosis usually required muscle biopsy confirmation. In the early 1990s, multiplex polymerase chain reaction provided a simple, rapid, and efficient alternative to identify common deletions. However, only selected exons were examined, and precise deletion borders could not be confirmed. In addition, duplication and small mutations could not be identified. Therefore, current diagnostic methods include multiplex ligationdependent probe amplification, comparative genomic hybridization microarray, or whole gene sequencing to detect deletions and duplications in all 79 exons [4].

Identification of a pathogenic point mutation in a DMD or BMD patient confirms the clinical diagnosis and allows definitive carrier testing and prenatal diagnosis for family members. Precise knowledge of the mutation is also required for some of the emerging therapies, such as exon skipping or suppression of premature stop codons [11].

Approximately $10-15 \%$ of DMD cases are caused by a nonsense mutation, a point mutation that causes a change in a triplet codon such that it no longer cods for an amino acid but instead cods for a stop signal. Interestingly, approximately $47 \%$ of patients with nonsense mutations can be potentially treated with exon skipping [12].

Exon skipping with antisense oligonucleotides (AON, morpholinos) is another gene- modifying technique trialed in patients with DMD. AON are short nucleic acid analogues that attach themselves to messenger RNA or single-stranded DNA and prevent its translation into a protein, effectively silencing part of a gene. In the context of DMD, AON have been designed to target mutation-containing exons in one of the hot-spots of the dystrophin gene. Normally, an out of frame mutation leads to a stop of dystrophin expression and the more severe DMD phenotype. However, by skipping the deleted or duplicated exon, the out of frame mutation is turned into an in frame mutation. Dystrophin expression can therefore continue at a reduced level, which results in the milder Becker muscular dystrophy. Drisapersen is an AON that cause skipping of exon51, which is a therapeutic strategy applicable to about $13 \%$ of boys with DMD. [13]

Another potential therapeutic approach for nonsense DMD mutations is stop codon read-through drugs such as Ataluren. They are orally delivered small molecules. Read-through drugs such as gentamicin, negamycin and ataluren (formerly known as PTC124) are reported to include ribosomal readthrough of premature stop codons, and restore dystrophin expression [12].

gentamicin induced read-through of the nonsense mutation TGA in dystrophin gene. The nonsense mutation TAA and TAG were not. Howard et al. reported that significant differences in the efficiency of aminoglycosideinduced read-through were observed, with UGA showing greater translational read-through than UAG or UAA, which coincides with our findings. Therefore, we cannot expect the same results of gentamicin treatment in all DMD patients who have stop codon mutations. It is speculated that this treatment is more effective for DMD patients with TGA stop codon than for those with the TAA and TAG stop codon [14].

Barton-Davis et al. demonstrated the possibility of treating DMD patients with nonsense mutations using aminoglycoside treatment. They use gentamicin to successfully suppress nonsense mutations and consequently restore dystrophin expression. Aminoglycoside antibiotic are currently being tested for efficacy in treatment of DMD patients carrying a nonsense mutation in the dystrophin gene. However, it is very difficult to identify DMD patients that are suitable for this therapy. In order to locate nonsense mutations, analyzing the $14 \mathrm{~kb}$ sequence of dystrophin cDNA is very time consuming and labor intensive. In addition, the efficiency of readthrough by gentamicin depends on the kind of nonsense mutation. In order for this therapy to be applicable, patients that may be treated with aminoglycoside therapy must first be readily identified [14]

This study was performed to evaluated point mutations in Iranian DMD/BMD male patients. A total of 29 DNA samples from patients who did not show any large deletion/duplication mutations following multiplex polymerase chain reaction (PCR) and multiplex ligation-dependent probe amplification (MLPA) screening were sequenced for detection of point mutations in exons 50_79. Cycle sequencing revealed four nonsense, one frame shift and two splice site mutations as well as two missense variant. We also introducing three novel variants.

\section{Acknowledgment}

We appreciate all who helped us to exert this study. 


\section{References}

[1] Beytía Mde L, Vry J, Kirschner J. Drug treatment of Duchenne muscular dystrophy: available evidence and perspectives. Acta Myol. 2012;31(1):4-8.

[2] Shimizu-Motohashi Y, Miyatake Sh, Komaki H, Takeda Sh, Aoki Y. Recent advances in innovative therapeutic approaches for Duchenne muscular dystrophy: from discovery to clinical trials. Am J Transl Res. 2016; 8(6): 24712489.

[3] S. Ryder, R. M. Leadley, N. Armstrong, M. Westwood, S. de Kock, T. Butt, et al.The burden, epidemiology, costs and treatment for Duchenne muscular dystrophy: an evidence review. Orphanet J Rare Dis. 2017; 12: 79

[4] Mah JK, Korngut L, Dykeman J, Day L, Pringsheim T, Jette N. A systematic review and meta-analysis on the epidemiology of Duchenne and Becker muscular dystrophy. Neuromuscul Disord. 2014;24(6):482-91.

[5] Bushby K, Finkel R, Birnkrant DJ, Case LE, Clemens PR, Cripe L, et al, Shapiro F, Tomezsko J, Constantin C; Diagnosis and management of Duchenne muscular dystrophy, part 1: diagnosis, and pharmacological and psychosocial management. Lancet Neurol. 2010;9(1):77-93

[6] Den Dunnen JT, Grootscholten PM, Bakker E, Blonden LA, Ginjaar HB, Wapenaar MC, van Paassen HM, et al. Topography of the Duchenne muscular dystrophy (DMD) gene: FIGE and cDNA analysis of 194 cases reveals 115 deletions and 13 duplications. Am J Hum Genet. 1989 Dec;45(6):835-47.

[7] Campbell KP. Three muscular dystrophies: loss of cytoskeleton-extracellular matrix linkage. Cell. 1995;80(5):675-9.

[8] Suthar R, Sankhyan N. Duchenne Muscular Dystrophy: A Practice Update. Indian J Pediatr. 2017 Jun 27

[9] Zimowski JG, Pawelec M, Purzycka JK, Szirkowiec W, Zaremba J. Deletions, not duplications or small mutations, are the predominante new mutations in the dystrophin gene. J Hum Genet. 2017;62(10):885-888.

[10] T Grimm, G Meng, S Liechti-Gallati, T Bettecken,C R Muller, B Muller. On The origin of deletions and point mutations in Duchenne Muscular dystrophy: most deletions arise in oogenesis and most point mutations result from events in spermatogenesis. J Med Genet 1994;31:183-186

[11] A Torella, A Trimarco, F Del Vecchio Blanco, A Cuomo, S Aurino, GPiluso, Carlo Minetti et al. One Hundred Twenty-One Dystrophin Point Mutations Detected from Stored DNA Samples by Combinatorial Denaturing HighPerformance Liquid Chromatography. J Mol Diagn. 2010; 12(1): 65-73.

[12] Yokota T, Duddy W, Echigoya Y, Kolski H. Exon skipping for nonsense mutations in Duchenne muscular dystrophy: too many mutations, too few patients? Expert Opin Biol Ther. 2012;12(9):1141-52.

[13] Straub V.Strehle EM, Recent advances in the management of Duchenne muscular dystrophy. Arch Dis Child. 2015 Dec;100(12):1173-7

[14] Kimura S, Ito K, Miyagi T, Hiranuma T, Yoshioka K, Ozasa S, Matsukura M, Ikezawa M, Matsuo M, Takeshima Y, Miike. A novel approach to identify Duchenne muscular dystrophy patients for aminoglycoside antibiotics therapy. Brain Dev. 2005;27(6):400-5 